\title{
Organic liquid-crystal devices based on ionic conductors
}

\section{Citation}

Yang, Can Hui, Shuang Zhou, Samuel Shian, David R. Clarke, and Zhigang Suo. 2017. “Organic Liquid-Crystal Devices Based on lonic Conductors." Materials Horizons 4 (6): 1102-1109. doi:10.1039/c7mh00345e.

\section{Published Version}

10.1039/C7MH00345E

\section{Permanent link}

http://nrs.harvard.edu/urn-3:HUL.InstRepos:34854277

\section{Terms of Use}

This article was downloaded from Harvard University's DASH repository, and is made available under the terms and conditions applicable to Open Access Policy Articles, as set forth at http:// nrs.harvard.edu/urn-3:HUL.InstRepos:dash.current.terms-of-use\#OAP

\section{Share Your Story}

The Harvard community has made this article openly available.

Please share how this access benefits you. Submit a story.

\section{Accessibility}




\section{Organic Liquid-Crystal Devices Based on Ionic Conductors}

Can Hui Yang, ${ }^{\text {a,b,c }}$ Shuang Zhou, ${ }^{\text {acc }}$ Samuel Shian, ${ }^{a}$ David R. Clarke ${ }^{\mathrm{a}}$ and Zhigang Suo* ${ }^{\mathrm{a}, \mathrm{c}}$

${ }^{a}$ John A. Paulson, School of Engineering and Applied Science, Harvard University, Cambridge, MA 02138, USA. E-mail: suo@seas.harvard.edu

${ }^{b}$ International Center for Applied Mechanics, State Key Laboratory for Strength and Vibration of Mechanical Structures, School of Aerospace, Xi'an Jiaotong University, Xi'an, 710049, China.

${ }^{c}$ Kavli Institute for Bionano Science and Technology, Harvard University, Cambridge, MA 02138, USA.

Liquid crystals have enabled many electrooptical devices, including liquid-crystal displays, ${ }^{1}$ universal optical phase modulators, ${ }^{2}$ beam steering devices, ${ }^{3}$ tunable Fresnel lenses, ${ }^{4,5}$ broad-spectrum tunable color reflectors, and nanosecond light modulators. ${ }^{6.7}$ These devices require transparent conductors to apply voltage and let light through. The widely used transparent conductor, indium tin oxide (ITO), is a brittle and costly inorganic, and requires vacuum for deposition. Enormous efforts are devoted to the development of new transparent conductors, usually using soft matrices to host conducting materials, such as conducting polymers, ${ }^{8-10}$ carbon nanotubes,,${ }^{11-13}$ graphene,,${ }^{14-16}$ nanoparticulate metal oxides, ${ }^{17}$ and silver nanowires. ${ }^{18-20}$

These conductors, like ITO, carry electricity using electrons. The development of transparent electronic conductors has many challenges. First, conduction electrons reflect or absorb light, so that electronic conductors cannot simultaneously achieve high electrical conductance and high optical transmittance. Second, most electronic conductors are hard inorganic materials, prone to fracture and delamination under monotonic and cyclic 
stretch. ${ }^{21-23}$ Patterning or pre-straining can make composite electrodes stretchable, but constituent materials remain mechanically mismatched. ${ }^{24-28}$ Third, devices requiring materials of very different processing conditions may cause additional technical issues for roll-to-roll fabrication and digital manufacturing. ${ }^{29,30}$ These challenges limit further development of electronic conductors for flexible and wearable devices, especially when large stretchability becomes essential.

Here we show that liquid-crystal devices can operate without electronic conductors, but with ionic conductors. We use polymer gels as transparent ionic conductors to realize fully organic liquid-crystal devices (OLCDs). They achieve the same electrooptical characteristics as liquid crystal devices using electronic conductors. The OLCDs perform electrooptical functions without electrochemical reactions. Furthermore, the polymer gels are stretchable, so that the OLCDs are optically responsive to both mechanical force and electrical voltage; they are mechanooptical and electrooptical devices.

Many polymer gels are stretchable, transparent, ionic conductors. ${ }^{31}$ For example, a hydrogel is a polymer network swollen with water, and can dissolve ions of multiple moles per liter, leading to resistivity $\sim 10^{-1} \Omega \mathrm{m}^{32}$ Even through this resistivity is much higher than that of ITO $\left(\sim 10^{-5} \Omega \mathrm{m}\right)$, a millimeter-thick hydrogel can achieve surface resistance of $100 \Omega$ and retain optical transparency of $99.9 \% .^{31}$ The softness of the gels allows large, elastic deformation over many cycles. ${ }^{31,33-37}$ The properties of hydrogels can be tuned by adjusting the cross-linkers. ${ }^{38-40}$ Hydrogels as tough as natural rubber have been demonstrated. ${ }^{41,42}$ Hydrogels can retain water in low-humidity environment if they contain humectants, ${ }^{32}$ or are encapsulated in low-permeability elastomers. ${ }^{43,44}$ As another example, an ionogel is a polymer 
network swollen with an ionic liquid. Ionogels are nonvolatile even in vacuum, ${ }^{35}$ and can access a wide range of temperature through suitable choices of ionic liquid. ${ }^{45}$ In addition, a nanostructured organic liquid crystal polymer electrolyte also achieves ionic conductivity on the order of $10 \Omega \mathrm{m}^{46}$

Recent works have demonstrated that ionic conductors can enable devices of unusual characteristics. Devices of high voltage $(10 \mathrm{kV}),{ }^{31,35}$ and high frequency (up to $\left.100 \mathrm{MHz}\right)^{47}$ have been demonstrated, as well as artificial muscle, axon, and skin. ${ }^{31,47-49}$ Potential applications include transparent loudspeakers and active noise cancelation, ${ }^{31}$ strain and pressure sensors, ${ }^{43,50-52}$ and stretchable touch pads. ${ }^{53}$ The soft devices can be made attachable to living tissues and soft robots. Ionic conductors have also enabled ionotronic devices, which integrate ionic conductors and electronic conductors. Examples include the rocking-chair operation of light-emitting diodes,${ }^{47}$ and stretchable electroluminescence. ${ }^{48,49,54}$

We now introduce ionic conductors in liquid-crystal devices. Whereas the principle applies to all liquid-crystal devices, here as a proof of concept we demonstrate a voltage-driven light shutter (Fig. 1). A cholesteric liquid crystal is encapsulated in a dielectric cell, sandwiched between ionic conductors, and connected via electronic conductors to an external voltage source. We do not treat the surfaces of the dielectric cell with mechanical rubbing or alignment agents, so that the liquid-crystal molecules orient randomly on the dielectric surfaces. When voltage is off, the cholesteric forms focal conic texture (twist domains with randomly orientated helical axis), the domain boundaries scatter light, and the device is opaque (Fig. 1a). When voltage is on, the electric field unwinds the twisted domains, the liquid-crystal molecules align into a homeotropic state, and the device is transparent (Fig. 
1b). The dielectric cell is made of an elastomer, and the ionic conductors are made of gels; they are transparent and stretchable. The electronic conductors are opaque and rigid metal foils; they contact the ionic conductors outside the area of the liquid crystals, and do not affect transmittance and stretchability of the device.

Each interface between an ionic conductor and an electronic conductor forms an electric double layer (EDL). As we will show below, the voltage drop over each EDL is much lower than $1 \mathrm{~V}$, so that the EDL remains stable, and behaves like a capacitor, $C_{E D L}$, in series with the capacitors due to the dielectric elastomers and the liquid crystals, $C_{D}$ and $C_{L C}$, as well as the resistors due to the ionic conductors, $R_{\text {gel }}$ (Fig. 1c).

We use three layers of an acrylic elastomer (VHB 4905, 3M) to make the dielectric cell, two layers of a polyacrylamide hydrogel containing $8.0 \mathrm{M}$ lithium chloride as the ionic conductors, and two pieces of aluminum as the electronic conductors (Fig. 2a). We form the cholesteric liquid crystal by mixing a nematic (5CB, $95.0 \mathrm{wt} \%)$ and a chiral dopant (R-811, $5.0 \mathrm{wt} \%$ ) (Fig. 2b). The cholesteric has the same thickness as the middle layer of the dielectric cell. When voltage is off, the area covered by the cholesteric is opaque, and the area covered by the hydrogel and elastomer is transparent (Fig. 2c). When voltage is on, the cholesteric becomes transparent (Fig. 2d). We apply alternating voltage to prevent space charges from accumulating at the interfaces between the cholesteric and the dielectric. Such space charges would build an internal electric field to counteract the applied electric field. ${ }^{1}$

After the voltage is switched on, the transmittance of the device increases with time and then saturates (Fig. 2e, Movie S1). The saturated transmittance increases as the voltage increases, and plateaus at large voltage (Fig. 2f). We measure the switch-on time $\tau_{\text {on }}$ and 
switch-off time $\tau_{\text {off }}$ (Fig. S1). ${ }^{1}$ The inverse of the measured switch-on time is proportional to the square of voltage, $\frac{1}{\tau_{o n}} \propto V_{0}^{2}$, and the switch-off time is nearly independent of voltage (Fig. 2g).

We next relate the observed electrooptical characteristics to fundamental principles. The applied voltage should be low enough to avert electrical breakdown in the cholesteric and the dielectric, and avert electrolysis of the gel. We estimate the voltage drop on each component by comparing their impedances. The resistance of the hydrogel is $R_{g e l} \sim 10^{2} \Omega$, given the thickness $\sim 1 \mathrm{~mm}$, length $\sim 1 \mathrm{~cm}$ and width $\sim 1 \mathrm{~cm}$, and resistivity $\sim 10^{-1} \Omega \mathrm{m}$. The capacitance per unit area of the EDL is $c_{E D L} \approx 10^{-1} \mathrm{Fm}^{-2},{ }^{55}$ the area of the EDL is $A_{E D L} \approx 10^{-4} \mathrm{~m}^{2}$, and the angular frequency is $\omega=2 \pi f=2000 \pi$, so that the impedance of the EDL is $\left|Z_{E D L}\right|=\frac{1}{c_{E D L} A_{E D L}} \approx 15$. The capacitance of the dielectric (VHB) is $C_{D}=\varepsilon_{V H B} \varepsilon_{0} A_{\text {active }} / d_{V H B} \approx 2.1 \times 10^{-9} F$, where $\varepsilon_{V H B} \approx 4.7$ is the dielectric constant of the $\mathrm{VHB},{ }^{56} \varepsilon_{0}=8.85 \times 10^{-12} \mathrm{Fm}^{-1}$ is the permittivity of vacuum, $d_{V H B} \sim 20 \mu \mathrm{m}$ is the thickness of VHB 4905 under a biaxial pre-stretch of $\sim 5$, and $A_{\text {active }} \sim 10^{-3} \mathrm{~m}^{2}$ is the area of active region containing the cholesteric, so that the impedance of the dielectric is $\left|Z_{D}\right|=\frac{1}{\omega C_{D}} \approx 7.65 \times 10^{4} \Omega$. The cholesteric has the same thickness and area as the VHB layer. Whereas the orientation of the liquid-crystal molecules are random in the voltage off state and the isotropic (or average) dielectric constant of $5 \mathrm{CB}$ is $\varepsilon_{5 C B}^{i s o} \approx 11$, we use $\varepsilon_{5 C B}^{\prime \prime} \approx 20$, the dielectric constant parallel to the director, for estimation, ${ }^{57}$ since the equilibrium state has liquid-crystal molecules parallel to the field. We estimate that 
$C_{C L C} \approx 8.85 \times 10^{-9} \mathrm{~F},\left|Z_{C L C}\right|=\frac{1}{\omega C_{C L C}} \approx 1.8 \times 10^{4} \Omega$. For the applied voltage of $600 \mathrm{~V}$, voltage drop across $\mathrm{EDL}$ is $\sim 52.5 \mathrm{mV}$, which is much lower than the threshold voltage for electrolysis of water. The voltage drop on hydrogel is $~ 350.4 \mathrm{mV}$. The main voltage drops are on the cholesteric, $\sim 63.1 \mathrm{~V}$, and on the dielectrics, $\sim 268.1 \mathrm{~V}$ for each VHB layer. The electric field in the dielectric is $\sim 13.4 \mathrm{MV} \mathrm{m}^{-1}$, which is much smaller than the electrical breakdown field of VHB $\left(\sim 100 \mathrm{MV} \mathrm{m}^{-1}\right) .{ }^{51}$ Similarly, the electric field in the cholesteric is below the electrical breakdown field of the cholesteric $\left.\left(\sim 10 \mathrm{MV} \mathrm{m}^{-1}\right)\right)^{58,59}$

The applied voltage should be high enough to switch the liquid-crystal molecules from the twisted domains to the homeotropic state. The threshold electric field is given by $E_{t h}=\frac{\pi^{2}}{p} \sqrt{\frac{K_{2}}{\Delta \varepsilon \varepsilon_{0}}} \cdot{ }^{57}$ For the $5 \mathrm{CB} / \mathrm{R}-811$ mixture, the twist elastic constant is $K_{2}=3 \times 10^{-12} \mathrm{~N}$, the dielectric anisotropy is $\Delta \varepsilon=13$, and the pitch is determined as $p=\frac{1}{H T P \cdot c} \approx 2 \mu m$, where the HTP $\sim 10 \mu \mathrm{m}^{-1}$ is the helical twisting power of $\mathrm{R}-811$ in $5 \mathrm{CB}$, and $c=5.0 \mathrm{wt} \%$ is the concentration of R-811. ${ }^{1}$ Consequently, the threshold electric field to unwind the cholesteric is $E_{t h} \sim 0.8 \mathrm{MV} \mathrm{m}^{-1}$. This estimate is consistent with our observation that the device becomes transparent at $350 \mathrm{~V}$, which gives $E \sim 1.84 \mathrm{MV} \mathrm{m}^{-1}$ in the cholesteric (Fig. $2 d)$.

Similar to the dynamic process of the Frederiks transition in liquid crystals, ${ }^{60,61}$ the switch-on time $\tau_{\text {on }}$ scales as $\frac{1}{\tau_{\text {on }}} \propto \frac{1}{\eta_{\text {twist }}}\left|\Delta \varepsilon \varepsilon_{0} E^{2}-\left(\frac{2 \pi}{p}\right)^{2} K_{2}\right|$, where $\eta_{\text {twist }}$ is the rotational viscosity of liquid crystal. A large electric field $E$ shortens the switch-on time. For the cholesteric of rotational viscosity $\eta_{\text {twist }} \sim 80 \mathrm{mPa} \mathrm{s},{ }^{1}$ the theoretical limit of switch on time 
can reach the order of $10^{-5} \mathrm{~s}$ when the electric field is just below the electrical breakdown field of the liquid crystal $10 \mathrm{MV} \mathrm{m}^{-1}$. The switch-on time in our experiment reaches $\sim 14 \mathrm{~ms}$ when the voltage is $1000 \mathrm{~V}$. A faster response can be achieved under a higher voltage theoretically; however, our experiment is limited by the timing loop of the data acquisition program.

Switching off is a field-independent process of several stages. When the voltage is switched off, the liquid-crystal molecules transit from the homeotropic state to the planar state, and then from the planar state to the focal conic state. The dynamics of the nucleation process depends on the impurities in the cholesteric and irregularities on the dielectric surface. As expected, the measured switch-off time is a constant independent of the voltage applied (Fig. 2f). For comparison, we fabricate a device by using ITO glass as the electrode and VHB with similar thickness $(\sim 20 \mu \mathrm{m})$ as the spacer. The switch-off time remains constant, $\sim 8.2 \mathrm{~ms}$, at various applied voltage (Fig. S2). The liquid-crystal devices using ionic conductors have similar electrooptical characteristics as those using electronic conductors.

The response time of the device is not limited by the resistivity of the hydrogels. The capacitors due to the dielectric elastomers, the liquid crystals and the EDLs are in series. The equivalent capacitance is dominated by the small capacitors of the dielectric and the liquid crystals, both of which are on the order of $10^{-10} \mathrm{~F}$; while the large capacitance due to the EDLs, which are on the order of $10^{-5} \mathrm{~F}$, is negligible. The resulting capacitance of the device $C$ is $\sim 10^{-10} \mathrm{~F}$. Resistance due to the hydrogel is $R_{g e l} \sim 10^{2} \Omega$. As a result, $R C$ delay of the device is small, $\tau_{R C} \sim 10^{-8} \mathrm{~s}$, and will not contribute to the observed switching time of the device. 
We confirm the change of the cholesteric texture as a function of voltage under a microscope with one linear polarizer placed before the device, and with or without an analyzer after the device (Fig. 3a). In the absence of voltage, cholesteric forms focal conic texture, showing a pitch of $\sim 2 \mu \mathrm{m}$ (Fig. 3b). After the voltage is applied, the cholesteric domains unwind to align with the electric field. Homeotropic regions nucleate and grow with time. The remaining twisted domains form loops and threads; they decrease in length and gradually disappear. When the voltage is switched off, the twisted domains re-form. The dynamic process is observed when the device is placed between two parallel polarizers (Fig. 3c, Movie S2). When the voltage is off, rotating the analyzer to be perpendicular to the polarizer (Fig. 3d) or removing the analyzer (Fig. 3e) does not affect the low intensity in the view, indicating a strong scattering of light by the focal conic domains. When the voltage is on, the device shows homogeneous black field of view with the analyzer in perpendicular orientation (Fig. 3d, Movie S3), or homogeneous bright field of view without analyzer (Fig. 3e, Movie S4), indicating that the homeotropic liquid-crystal molecules do not scatter or modulate the polarization of light.

We next demonstrate a stretchable electrooptical device (Fig. 4, Fig. S3). We apply a pre-stretch of $\sim 1.3$ to the VHB before fabricating the device, and regard this as the reference state. In the reference state, the thickness of the cholesteric is $d \approx 296 \mu \mathrm{m}$. Let $\lambda$ be the stretch (i.e., the diameter of the device in the stretched state divided by that in the reference state). The device remains switchable at both the reference state $\lambda=1.0$ (Fig. 4a) and in the stretched state $\lambda=1.5$ (Fig. $4 \mathrm{~b}$ ). 
We also note a new mode of operation: the light shutter is switchable in response to a combined electrical voltage and mechanical force (Fig. 4c). When the device in the reference state is subject to a voltage of $1200 \mathrm{~V}$, the active area remains opaque. Then when the device is stretched to a large area, without changing the voltage, the active area becomes transparent (Movie S5). Since the transmittance as a function of electric field is steep, such an electromechanical light shutter switch on and off at narrow range of voltage and stretch. When the device is pulled by an equal-biaxial stretch $\lambda$, the thickness of the device reduces by a factor of $\lambda^{2}$, and the switching voltage is also reduced by a factor of about $\lambda^{2}$ (Fig. $4 \mathrm{~d}$ ). The inset shows that the relation between the transmittance and the product $V_{0} \lambda^{2}$ remains nearly the same for different values of $\lambda$.

The small difference between the three curves in the inset of Fig. $4 d$ is probably due to the elastic deflection of the dielectric and the hydrodynamic flow of the cholesteric, driven by the Maxwell stress (Fig. S4, Movie S6). This elastohydrodynamic-electrooptical coupling can be minimized by reducing the radius of the dielectric cell. The elastohydrodynamic-electrooptical coupling can, of course, enable devices of new functions, but we will not pursue this possibility here.

When the device is stretched, the same applied voltage can switch the device much faster. Since thinning of the cholesteric increases the electric field by a factor of $\lambda^{2}$, we expect $1 / \tau_{\text {on }}$ to increase by a factor of $V_{0}^{2} \lambda^{4}$. In the experiments, $\tau_{\text {on }}$ does show a significant decrease as $V_{0}$ or $\lambda$ increases (Fig. 4e), although $1 / \tau_{\text {on }}$ does not strictly follow a linear relationship with $V_{0}^{2} \lambda^{4}$ (Fig. 4e, inset), especially when the voltage is higher or the stretch is larger. The deviation is also likely caused by the elastohydrodynamic effect. 
The dielectric layers serve two functions. First, the dielectric layers prevent the mixing of the gel and the liquid crystal. Second, the dielectric layers prevent electrical breakdown even if the Maxwell stress causes the top and bottom surfaces to deflect into contact. The voltage in our experiment is high, but can be reduced by reducing the thickness of the dielectric and liquid crystal layers. One may even remove the dielectric and allow the liquid crystal to be in direct contact of the gels, as long as the gels and the liquid crystal are immiscible. The Maxwell stress will deflect the gels negligibly if the device is sandwiched between elastomers of suitable stiffness.

Lithium chloride also serves two functions. First, lithium chloride provides mobile ions after dissolving in water. Second, lithium chloride is a potent humectant. Whereas a hydrogel containing sodium chloride hydrogel dries out easily, the hydrogel containing $8.0 \mathrm{M}$ lithium chloride can retain water at relative humidity as low as $11 \% .^{32}$ The device maintains its switching performance well even after being exposed in the open air for more than six months. For critical conditions such as vacuum environment and temperature below freezing point or boiling point of hydrogel, one can of course use ionogels instead.

\section{Conclusions}

We have used ionic conductors to realize organic liquid-crystal devices (OLCDs). Liquid crystals are anisotropic dielectrics. Their electrooptical effects require application of voltage, not injection of electrons. Consequently, ionic conductors can be used to drive liquid crystal devices of all kinds. The basic structure of the devices is compatible with surface patterning, distribution of particles, polymer-dispersed liquid crystals, and polymer-stabilized 
liquid crystals. Ionic conductors can also drive arrays of pixels. OLCDs are fully organic, amenable to roll-to-roll and digital manufacturing.

The use of ionic conductors greatly expands the choice of transparent conductors. This large pool of materials will enable liquid-crystal devices of new characteristics. For example, one may choose a gel, an elastomer, and a liquid crystal to match their refractive indices, and thereby minimize the Fresnel reflections. By contrast, conventional liquid-crystal devices have mismatched reflective indices ( $~ 2.0$ for ITO, and $\sim 1.5$ for glass).

The new attribute of OLCDs - the softness - may bring liquid crystals to new domains of applications, such as wearable displays for camouflage, and curved, deformable displays for entertainment. One can also imagine programmable, high-resolution, tissue-attachable patterns of light for optogenetics and optoceuticals - that is, a direct TV for the brain, heart, and skin.

\section{Experimental Section}

\section{Synthesis of the ionic conductor}

The ionic conductor used in our experiment was a polyacrylamide hydrogel containing lithium chloride. To prepare the ionic conductor, we dissolved $2.4888 \mathrm{~g}$ of acrylamide (0.035 mol) and $5.94 \mathrm{~g}$ of lithium chloride $(0.14 \mathrm{~mol})$ in $17.5112 \mathrm{~g}$ of deionized water $(0.973 \mathrm{~mol})$. The resulting concentration of lithium chloride was $8.0 \mathrm{M}$. We then added to the solution $0.0015 \mathrm{~g}$ of crosslinker ( $\mathrm{N}, \mathrm{N}^{\prime}$-methylenebisacrylamide, MBAA, $\left.10^{-5} \mathrm{~mol}\right), 8 \mu \mathrm{L}$ of accelerator (N,N,N',N'-tetramethylethylenediamine, TEMED, $5.3 \times 10^{-5} \mathrm{~mol}$ ), and $0.0025 \mathrm{~g}$ of initiator (ammonium persulphate, APS, $1.1 \times 10^{-5} \mathrm{~mol}$ ). After careful stirring, we transferred the 
solution into a glass mold with a $1 \mathrm{~mm}$ thick spacer, and placed the solution in a fume hood to cure at room temperature for 6 hours. The as-prepared ionic conductor was removed from the mold, cut into pre-designed shape and then transferred onto the target device.

\section{Preparation of the cholesteric liquid crystal}

We made a cholesteric liquid crystal by mixing a nematic liquid crystal (4-Cyano-4'-pentylbiphenyl, 5CB) and a chiral liquid crystal (4-(hexyloxy)-, 4-[[[(1r)-1-methylheptyl]oxy]carbonyl], R-811) at a weight ratio of 95:5. The solution was homogenized using a vortex mixer (Digital Vortex Mixer, VWR) for $10 \mathrm{~min}$.

\section{Fabrication of electrooptical devices (Fig. S5)}

A layer of dielectric elastomer (VHB 4905, 3M) was cut into a disk with a hole at the center using a laser cutter (UNIVERSAL), followed by laminating onto another layer of VHB 4905. The two layers were fastened on a biaxial stretcher, pre-stretched and mounted on a rigid circular-ring frame. The cholesteric liquid crystal was added into the hole. Another layer of VHB 4905 with the same pre-stretch was laminated to seal the liquid crystal, forming a liquid-crystal cell. The polyacrylamide hydrogel was cut into circular shape with a tail for electrical connection, and then laminated on the two sides of the liquid-crystal cell. Two aluminum foils were placed on the hydrogels outside the active area to connect the device to the voltage source.

\section{Electrooptical measurements}


A signal generator (KEYSIGHT, 33500B) was used to supply square-wave signal at $1 \mathrm{kHz}$. The signal from the generator was fed through an amplifier (TRek, MODEL 30/20A) with a magnifying factor of 3000 to the two aluminum foils on the electrooptical device. The resulting voltage waveform had minimum and maximum amplitude of $-V_{0}$ and $+V_{0}$, respectively, where $V_{0}$ was the set voltage. Optical transmittance was measured by placing the device between two collimating lenses, connected using optical fibers to a tungsten light source and a spectrometer (Ocean Optics, USB 2000+). Before the measurement, a voltage was applied for $5 \mathrm{~s}$ and removed to obtain an initial opaque state. Data were collected with a custom-made LabVIEW program.

\section{Microscopic observation (Fig. S6)}

The device was observed under a microscope (Leica, DM 4000M) with one linear polarizer placed before the device, and with or without an analyzer after in either perpendicular or parallel orientation. A mobile phone was mounted on the ocular to record the dynamic process of the liquid-crystal device.

\section{Acknowledgements}

The work is supported by NSF MRSEC (DMR 14-20570) and NSF CMMI-1404653. C. H. Yang was supported by the China Scholarship Council as a visiting scholar at Harvard University.

\section{References}


1 D.-K. Yang, S.-T. Wu, Fundamentals of Liquid Crystal Devices, John Wiley \& Sons, Chichester, England, 2006.

2 R. Oldenbourg, Polarization Microscopy with the LC-Polscope, Cold Spring Harbor Laboratory Press, Cold Spring Harbor, 2005.

3 P. F. McManamon, J. Shi, P. J. Bos, Opt. Eng., 2005, 44, 128004.

4 O. Pishnyak, S. Sato, O. D. Lavrentovich, Appl. Opt., 2006, 45, 4576.

5 S. Susumu, S. Akira, S. Rumiko, Jpn. J. Appl. Phys., 1985, 24, L626.

6 S.-Y. Lu, L.-C. Chien, Opt. Lett., 2010, 35, 562.

7 V. Borshch, S. V. Shiyanovskii, O. D. Lavrentovich, Phys. Rev. Lett., 2013, 111, 107802.

8 P. A. Levermore, R. Jin, X. Wang, L. Chen, D. D. C. Bradley, J. C. de Mello, J. Mater. Chem., 2008, 18, 4414.

9 J. Huang, X. Wang, A. J. deMello, J. C. de Mello, D. D. C. Bradley, J. Mater. Chem., 2007, 17, 1043.

10 S.-I. Na, S.-S. Kim, J. Jo, D.-Y. Kim, Adv. Mater., 2008, 20, 4061.

11 Z. Chen, J. W. F. To, C. Wang, Z. Lu, N. Liu, A. Chortos, L. Pan, F. Wei, Y. Cui, Z. Bao, Adv. Energy Mater., 2014, 4, 1400207.

12 D. Chen, J. Liang, Q. Pei, Sci. China Chem., 2016, 59, 659.

13 D. McCoul, W. Hu, M. Cao, V. Mehta, Q. Pei, Adv. Electron. Mater., 2016, 2, 1500407.

14 K. S. Kim, Y. Zhao, H. Jang, S. Y. Lee, J. M. Kim, K. S. Kim, J.-H. Ahn, P. Kim, J.-Y.

Choi, B. H. Hong, Nature, 2009, 457, 706.

15 G. Eda, M. Chhowalla, Adv. Mater., 2010, 22, 505. 
16 P. H. Wöbkenberg, G. Eda, D.-S. Leem, J. C. de Mello, D. D. C. Bradley, M. Chhowalla, T. D. Anthopoulos, Adv. Mater., 2011, 23, 1558.

17 S. Yodyingyong, Q. Zhang, K. Park, C. S. Dandeneau, X. Zhou, D. Triampo, G. Cao, Appl. Phys. Lett., 2010, 96, 073115.

18 P. Blake, P. D. Brimicombe, R. R. Nair, T. J. Booth, D. Jiang, F. Schedin, L. A.

Ponomarenko, S. V. Morozov, H. F. Gleeson, E. W. Hill, A. K. Geim, K. S. Novoselov, Nano Letters, 2008, 8, 1704.

19 J. Liang, L. Li, X. Niu, Z. Yu, Q. Pei, Nature Photonics, 2013, 7, 817.

20 S. Shian, D. R. Clarke, Optics Lett., 2016, 41, 1289.

21 G. P. Crawford, Flexible Flat Panel Displays, John Wiley \& Sons, Chichester, England, 2005.

22 J. Lewis, Mater. Today, 2006, 9, 38.

23 K. Alzoubi, M. M. Hamasha, S. Lu, B. Sammakia, J. Display Technol., 2011, 7, 593.

24 S. P. Lacour, S. Wagner, Z. Huang, Z. Suo, Appl. Phys. Lett., 2003, 82, 2404.

25 D. J. Lipomi, M. Vosgueritchian, B. C-K. Tee, S. L. Hellstrom, J. A. Lee, C. H. Fox, Z. Bao, Nat. Nano., 2011, 6, 788.

26 M. L. Hammock, A. Chortos, B. C. K. Tee, J. B. H. Tok, Z. Bao, Adv. Mater., 2013, 25, 5997.

27 S. Yang, E. Ng, N. Lu, Extreme Mechanics Letters, 2015, $2,37$.

28 S. Yao, Y. Zhu, Adv. Mater., 2015, 27, 1480.

29 F. C. Krebs, Organic Electronics, 2009, 10, 1636. 
30 R. Søndergaard, M. Hosel, D. Angmo, T. T. Larson-Olsen, F. C. Krebs, Mater.Today, $2012, \mathbf{1 5}, 36$.

31 C. Keplinger, J. Y. Sun, C. C. Foo, P. Rothemund, G. M. Whitesides, Z. Suo, Science, 2013, 341, 984.

32 Y. Bai, B. Chen, F. Xiang, J. Zhou, H. Wang, Z. Suo, Appl. Phys. Lett., 2014, 151903.

33 A. K. Denisin, B. L. Pruitt, ACS Appl. Mater. Interfaces, 2016, 8, 21893.

34 P. Lin, T. Zhang, X. Wang, B. Yu, F. Zhou, Small, 2016, 12, 4386.

35 B. Chen, J. J. Lu, C. H. Yang, J. H. Yang, J. Zhou, Y. M. Chen, Z. Suo, ACS Appl. Mater. Interfaces, 2014, 6, 7840.

36 J. Tang, J. Li, J. J. Vlassak, Z. Suo, Extreme Mechanics Lett. 2017, 10, 24.

37 R. Bai, Q. Yang, J. Tang, X. P. Morelle, J. J. Vlassak, Z. Suo, Extreme Mechanics Lett. $2017, \mathbf{1 5}, 91$.

38 A. Southan, M. Mateescu, V. Hagel, M. Bach, C. Schuh, C. Kleinhans, P. J. Kluger, S. Tussetschlager, I. Nuss, T. Haraszti, S. V. Wegner, J. P. Spatz, H. Boehm, S. Laschat, G. E. M. Tovar, Macromol. Chem. Phys., 2013, 214, 1865.

39 V. Hagel, M. Mateescu, A. Southan, S. V. Wegner, I. Nuss, T. Haraszti, C. Kleinhans, C. Schuh, J. P. Spatz, P. J. Kluger, M. Bach, S. Tussetschlager, G. E. M. Tovar, S. Laschat, H. Boehm, Sci. Rep., 2013, 3, 2043.

40 M. Martini, P. S. Hegger, N. Schadel, B. B. Minsky, M. Kirchhof, S. Scholl, A. Southan, G. E. M. Tovar, H. Boehm, S. Laschat, Materials, 2016, 9, 810.

41 J. P. Gong, Y. Katsuyama, T. Kurokawa, Y. Osada, Adv. Mater., 2003, 15, 1155. 
42 J. Y. Sun, X. Zhao, W. R. K. Illeperuma, O. Chaudhuri, K. H. Oh, D. J. Mooney, J. J. Vlassak, Z. Suo, Nature, 2012, 489, 133.

43 J. Y. Sun, C. Keplinger, G. M. Whitesides, Z. Suo, Adv. Mater., 2014, 26, 7608.

44 H. Yuk, T. Zhang, G. A. Parada, X. Liu, X. Zhao, Nat. Commun., 2016, 7, 12028.

45 J. Le Bideau, L. Viau, A. Vioux, Chem. Soc. Rev., 2011, 40, 907.

46 R. L. Kerr, J. P. Edwards, S. C. Jones, B. J. Elliott, D. L. Gin, Poly. J., 2016, 48, 635.

47 C. H. Yang, B. Chen, J. J. Lu, J. H. Yang, J. Zhou, Y. M. Chen, Z. Suo, Extreme Mechanics Letters, 2015, 3, 59.

48 C. Larson, B. Peele, S. Li, S. Robinson, M. Totaro, L. Beccai, B. Mazzolai, R. Shepherd, Science, 2016, 351, 1071.

49 C. H. Yang, B. Chen, J. Zhou, Y. M. Chen, Z. Suo, Adv. Mater., 2016, 28, 4480.

50 S. S. Robinson, K. W. O’Brien, H. Zhao, B. N. Peele, C. M. Larson, B. C. M. Murray, I. M. V. Meerbeek, S. N. Dunham, R. F. Shepherd, Extreme Mechanics Letters, 2015, 5, 47.

51 P. Manandhar, P. D. Calvert, J. R. Buck, IEEE Sensors Jour., 2012, 12, 2052.

52 J. Guo, X. Liu, N. Jiang, A. K. Yetisen, H. Yuk, C. Yang, A. Khademhosseini, X. Zhao, S.-H. Yun, Adv. Mater., 2016, 28, 10244.

53 C. C. Kim, H. H. Lee, K. H. Oh, J. Y. Sun, Science, 2016, 353, 682.

54 J. Wang, C. Yan, G. Cai, M. Cui, A. L. S. Eh, P. S. Lee, Adv. Mater., 2016, 28, 4490.

55 K. H. Lee, M. S. Kang, S. Zhang, Y. Gu, T. P. Lodge, C. D. Frisbie, Adv. Mater., 2012, 24, 4457.

56 B. Chen, Y. Bai, F. Xiang, J.Y. Sun, Y.M. Chen, H. Wang, J. Zhou, Z. Suo, J. Polym. Sci., Part B: Polym. Phys., 2014, 52, 1055. 
57 M. Kleman, O. D. Lavrentovich, Soft Matter Physics: An Introduction, Springer, New York, 2003.

58 R. B. Meyer, Appl. Phys. Lett., 1968, 12, 281.

59 J. J. Wysocki, J. Adams, W. Haas, Phys. Review Lett., 1968, 20, 1024.

60 A. J. Bard, L. R. Faulkner, Electrochemical Methods: Fundamentals and Applications, John Wiley \& Sons, New York, 2000.

61 P. G. de Gennes, J. Prost, The Physics of Liquid Crystals, Clarendon Press, Oxford, 1993. 


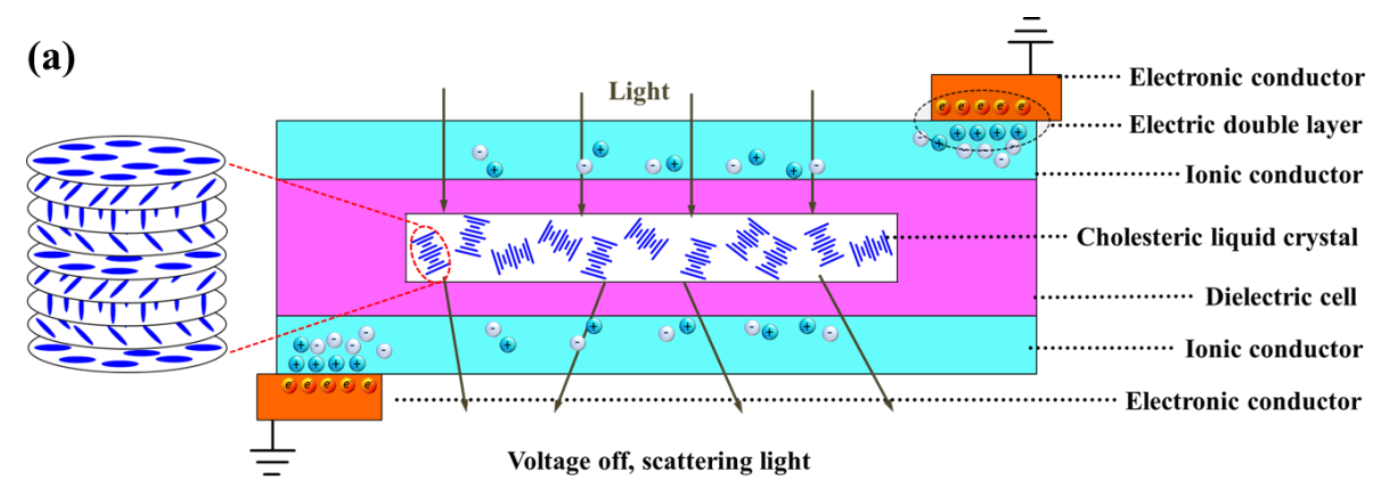

(b)
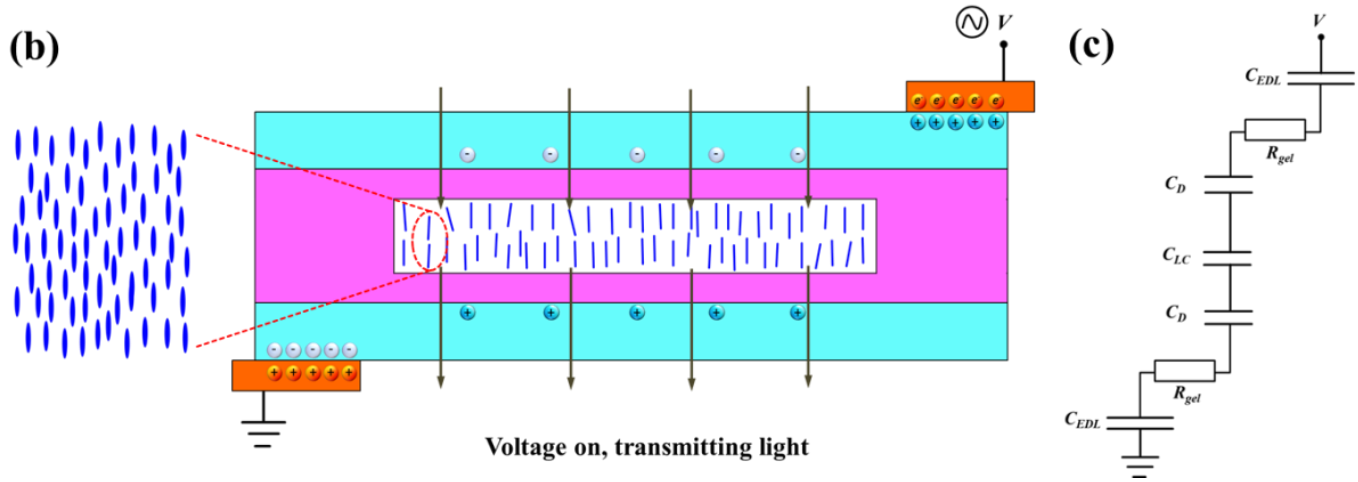

Fig. 1 Organic liquid-crystal device based on ionic conductors. (a) A cholesteric liquid crystal is encapsulated in a dielectric cell, sandwiched between ionic conductors, and connected via electronic conductors to an external voltage source. When the external voltage is off, the liquid crystal molecules form twisted domains, the domain boundaries scatter light, and the device is opaque. (b) When the external voltage is on, the domains unwind, the molecules align with the electric field, and the device is clear. (c) Equivalent circuit of the device. 
(a)

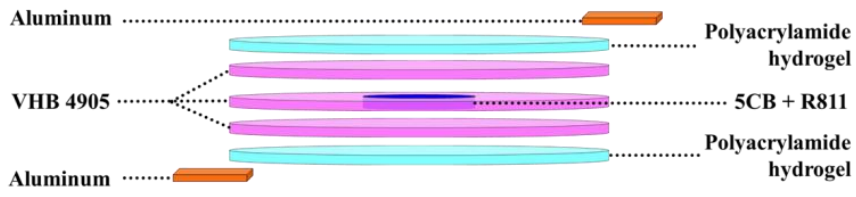

(b)

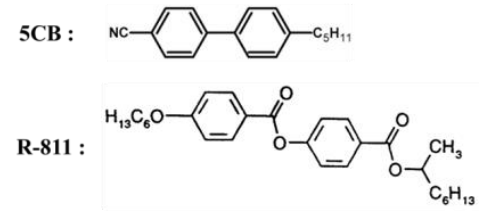

(c)

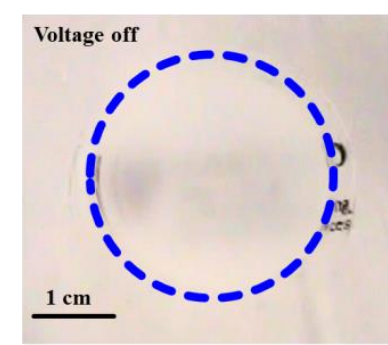

(f)

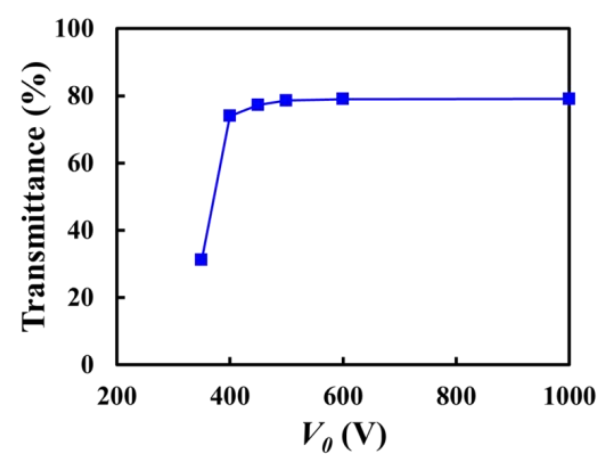

(d)

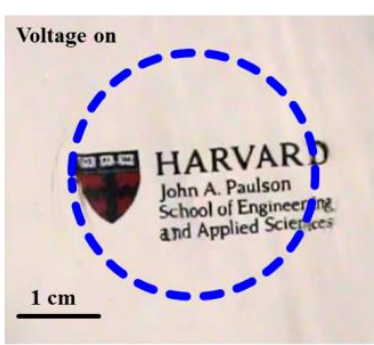

(g)
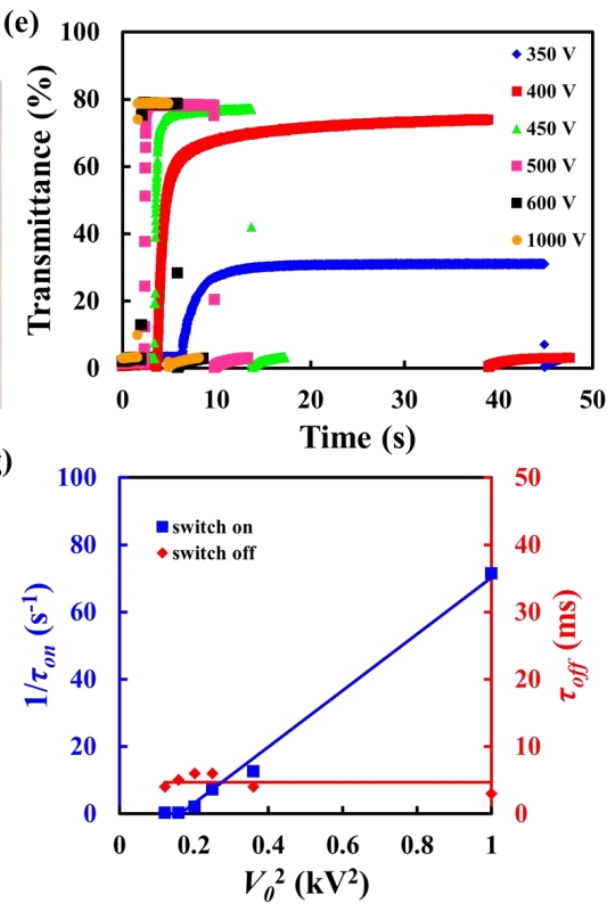

Fig. 2 Electrooptical characteristics. (a) Schematic of a liquid-crystal device. (b) Molecular structures of nematic liquid crystal 5CB and chiral dopant R-811. (c) When voltage is off, the device is opaque. (d) When voltage is on, the device is transparent. A square-wave voltage is applied at amplitude $600 \mathrm{~V}$ and frequency $1 \mathrm{kHz}$. The dashed circles indicate the areas containing the liquid crystal. (e) Transmittance of the device as a function of time under square-wave voltage of various amplitudes at a frequency of $1 \mathrm{kHz}$. (f) The transmittance of the device increases and then saturates as voltage increases. (g) $1 / \tau_{\text {on }}$ increases linearly with the voltage square, and $\tau_{\text {off }}$ is almost constant. The solid lines are linear-fitting curves. 
(a)

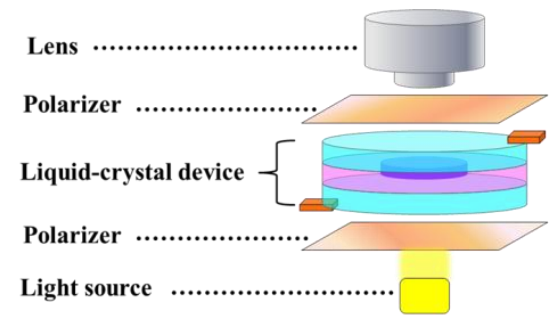

(c)
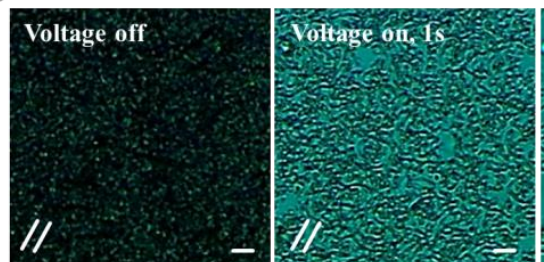

(d)

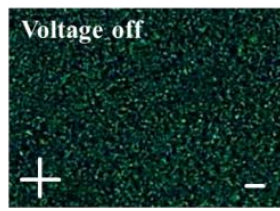

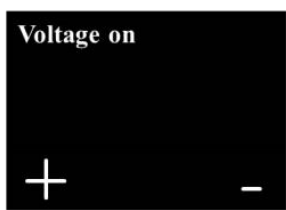

(e)

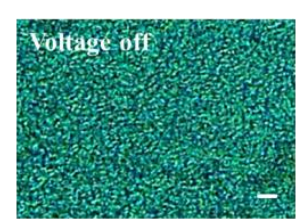

(b)
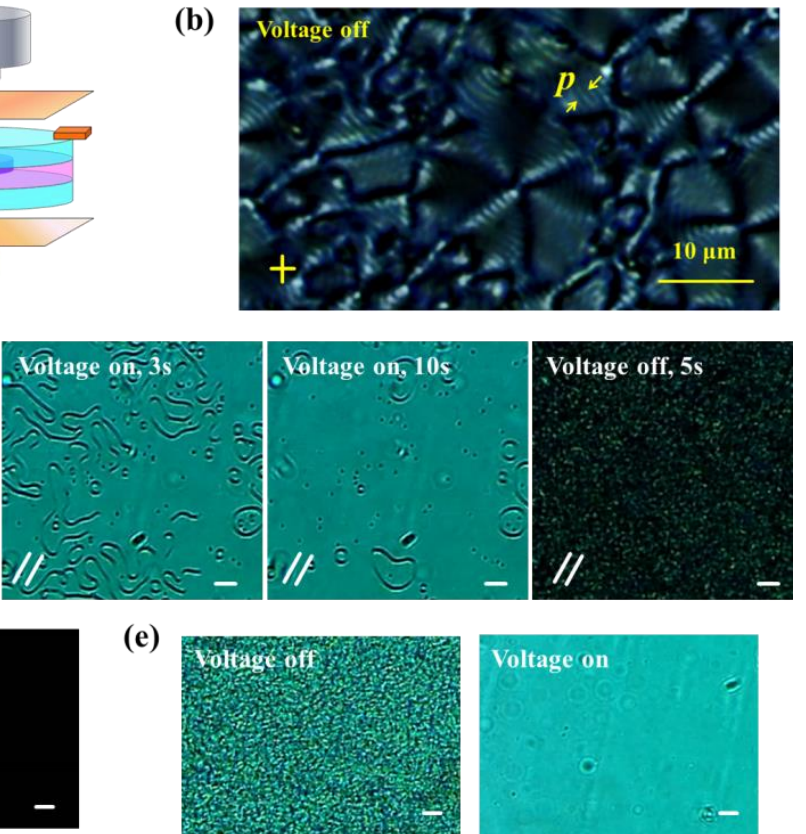

Fig. 3 Voltage-dependent microstructures. (a) Schematic of experimental setup. (b) Focal conic texture of the cholesteric placed between perpendicular polarizers, observed at the voltage-off state. (c) Dynamic process of the cholesteric observed with parallel polarizers (Movie S2) at voltage of amplitude $500 \mathrm{~V}$ and frequency $1 \mathrm{kHz}$. (d) Device placed between perpendicular polarizers, observed at the voltage-off and voltage-on states (Movie S3). (e) Device placed in the microscope without analyzer, observed at the voltage-off and voltage-on states (Movie S4). Scales bars in (c), (d) and (e) indicate $100 \mu \mathrm{m}$. 
(a)

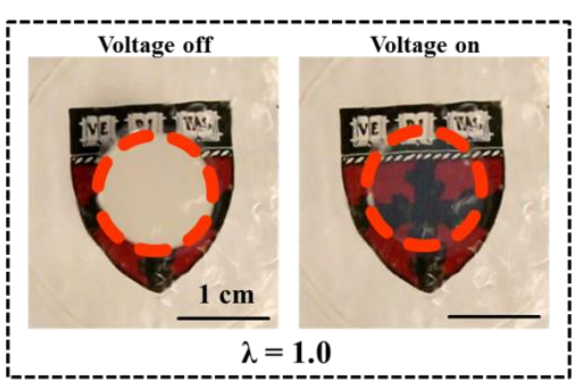

(b)

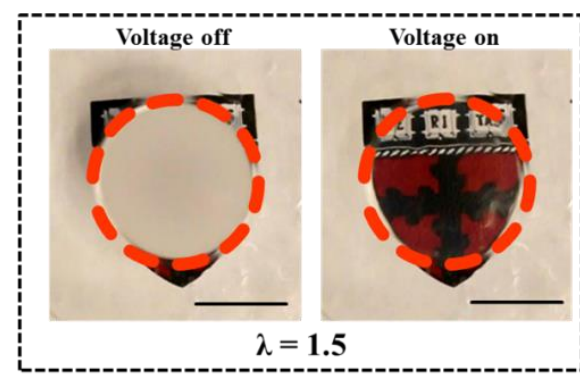

(c)

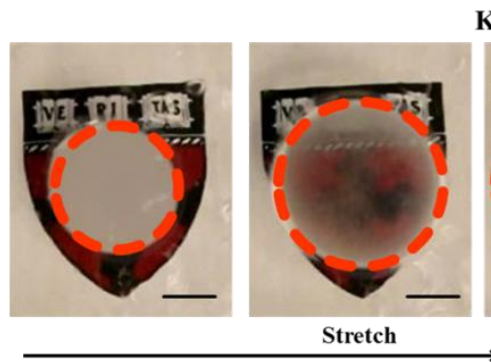

Keep voltage on

(d)

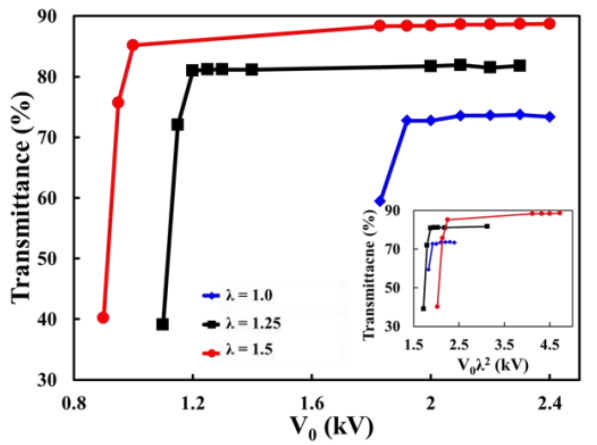

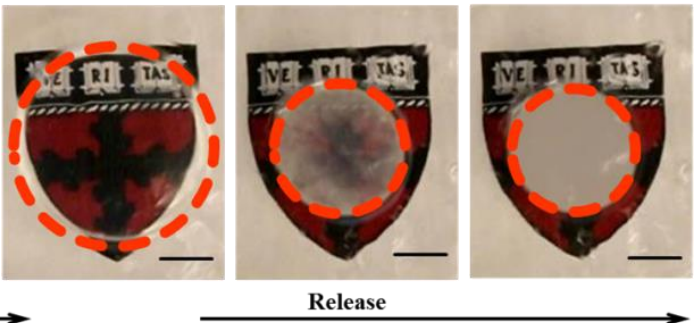

(e)

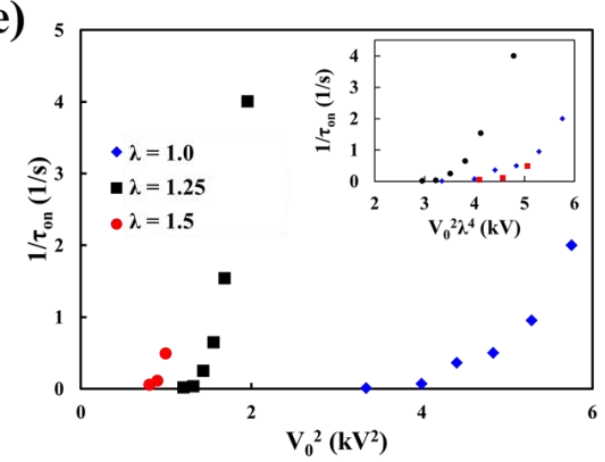

Fig. 4 Stretchable liquid-crystal device. (a) The device is switched in the undeformed state, $\lambda=1$.0. (b) The device is switched in the stretched state, $\lambda=1.5$. The dashed circles indicate the area containing cholesteric. (c) The transparency changes in a cycle of stretching and releasing. A square-wave voltage of amplitude $1200 \mathrm{~V}$ and frequency $1 \mathrm{kHz}$ is applied during the whole process. (d) The transmittance as a function of voltage at several values of stretch. The maximum transmittance increases while the threshold voltage for switching decreases as $\lambda$ increases. Inset: the transmittance as a function of electric field intensity. (e) $1 / \tau_{o n}$, as a function of voltage squared at various $\lambda$. Inset: $1 / \tau_{\text {on }}$ as a function of electric field squared. 